\title{
Ganho de peso gestacional excessivo no Sistema Único de Saúde
}

Excessive gestational weight gain in the Brazilian Unified Health System Aumento de peso gestacional excesivo en el Sistema Único de Salud

\author{
Lorenna Viccentine Coutinho Monteschio ${ }^{1}$ (D https://orcid.org/0000-0002-1486-6898 \\ Sonia Silva Marcon ${ }^{1}$ iD https://orcid.org/0000-0002-6607-362X \\ Guilherme Oliveira de Arruda ${ }^{2}$ id https://orcid.org/0000-0003-1690-4808 \\ Elen Ferraz Teston ${ }^{3}$ (D https://orcid.org/0000-0001-6835-0574 \\ Evelin Matilde Arcain Nass ${ }^{1}$ io https://orcid.org/0000-0002-5140-3104 \\ Josane Rosenilda da Costa ${ }^{1}$ iD https://orcid.org/0000-0003-0369-977X \\ Mônica Oliveira Batista Oriá ${ }^{4}$ (D) https://orcid.org/0000-0002-1483-6656 \\ Adriana Lenho de Figueiredo Pereira ${ }^{5}$ id https://orcid.org/0000-0002-2563-6174
}

Monteschio LV, Marcon SS, Arruda GO, Teston

EF, Nass EM, Costa JR, et al. Ganho de peso

gestacional excessivo no Sistema Único de

Saúde. Acta Paul Enferm. 2021;34:eAPE001105.

DOI

http://dx.doi.org/10.37689/actaape/2021A0001105

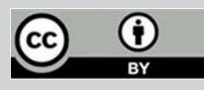

Descritores

Ganho de peso; Gravidez; Alterações do peso corporal; Saúde da mulher; Atenção primária à

saúde

Keywords

Body weight changes; Pregnancy; Primary health care; Weight gain; Women's health

Descriptores

Atención primaria de salud; Aumento de peso; Cambios en el peso corporal; Embarazo; Salud

de la mujer

Submetido

12 de Maio de 2020

Aceito

23 de Março de 2021

Autor correspondente

Lorenna Viccentine Coutinho Monteschio

E-mail: helorenn@gmail.com

\section{Resumo}

Objetivo: Verificar a prevalência e os fatores associados ao ganho de peso excessivo na gestação.

Métodos: Estudo transversal, realizado em município do interior do sul do Brasil, com 462 mulheres que tiveram parto financiado pelo Sistema Único de Saúde. Coletado dados sociodemográficos, antropométricos, obstétricos, hábitos alimentares e prática de atividade física. Realizou-se análise estatística bivariada (Quiquadrado) e múltipla por meio de modelo de regressão logística.

Resultados: Participaram deste estudo, predominantemente, mulheres casadas/ em união estável, maiores de 25 anos e das classes econômicas C, D ou E. A prevalência de ganho de peso excessivo na gestação foi de $38,3 \%$. As mulheres com renda per capita menor que um salário-mínimo apresentaram menor frequência de ganho de peso excessivo na gestação $(p=0,020)$. Já as gestantes que referiram planejar a gestação $(p=0,048)$, que tinham excesso de peso pré-gestacional $(p<0,001)$, que aumentaram a ingesta alimentar $(p<0,001)$ e que consumiram produtos industrializados mais do que três vezes por semana $(p=0,002)$ foram as que apresentaram maior frequência de ganho de peso excessivo.

Conclusão: A prevalência de ganho de peso gestacional excessivo foi de 38,3\% e esteve associada à maior renda per capita, gestação planejada, excesso de peso pré-gestacional, aumento da ingesta alimentar e maior frequência semanal no consumo de produtos industrializados.

\section{Abstract}

Objective: To check the prevalence and factors associated with excessive weight gain during pregnancy.

Methods: This is a cross-sectional study carried out in a municipality in the countryside of southern Brazil, with 462 women who had a birth financed by the Brazilian Unified Health System. Sociodemographic, anthropometric, obstetric, eating habits and physical activity data were collected. Bivariate (chi-square) and multiple statistical analysis were performed using a logistic regression model.

Results: The study was predominantly carried out by women who were married/in a stable relationship, over 25 years of age and from economic classes C, D or E. The prevalence of excessive weight gain during pregnancy was $38.3 \%$. Women with per capita income below one minimum wage had a lower frequency of excessive weight gain during pregnancy $(p=0.020)$. The pregnant women who reported planning their pregnancy $(p=0.048)$, who were overweight pre-pregnancy $(p<0.001)$, who increased their food intake $(p$ $<0.001)$ and who consumed industrialized products more than three times a week $(p=0.002)$ were those that presented a higher frequency of excessive weight gain.

'Universidade Estadual de Maringá, Maringá, PR, Brasil.

¿Universidade Federal do Mato Grosso do Sul, Coxim, MS, Brasil.

Universidade Federal do Mato Grosso do Sul, Campo Grande, MS, Brasil.

${ }^{4}$ Universidade Federal do Ceará, Fortaleza, CE, Brasil.

Eniversidade do Estado do Rio de Janeiro, Rio de Janeiro, RJ, Brasil.

Conflitos de interesse: extraído da tese: "Prevalência e fatores associados ao ganho de peso gestacional excessivo e retenção de peso pós-parto" apresentada ao

Programa de Pós-graduação em Enfermagem da Universidade Estadual de Maringá (UEM), 2019. 
Conclusion: The prevalence of gain of excessive gestational weight was 38.3\% and was associated with higher per capita income, planned pregnancy, pregestational excess weight, increased food intake and higher weekly frequency of consumption of industrialized products.

\section{Resumen}

Objetivo: Verificar la prevalencia y los factores asociados al aumento de peso excesivo en la gestación.

Métodos: Estudio transversal, realizado en un municipio del interior de la región Sur de Brasil, con 462 mujeres cuyo parto fue financiado por el Sistema Único de Salud. Se recopilaron datos sociodemográficos, antropométricos, obstétricos, hábitos alimentarios y práctica de actividad física. Se realizó un análisis estadístico bivariado (ji cuadrado) y múltiple mediante el modelo de regresión logística.

Resultados: Participaron en este estudio principalmente mujeres casadas/con unión de hecho, mayores de 25 años y de clase económica C, D o E. La prevalencia del aumento de peso excesivo en la gestación fue del 38,3 \%. Las mujeres con ingreso per cápita menor a un salario mínimo presentaron menor frecuencia de aumento de peso excesivo en la gestación $(\mathrm{p}=0,020)$. Por otro lado, las mujeres embarazadas que indicaron una gestación planificada $(\mathrm{p}=0,048)$, que tenían exceso de peso pregestacional $(p<0,001)$, que aumentaron la ingesta de alimentos $(p<0,001)$ y que consumieron productos industrializados más de tres veces por semana ( $p=0002)$ fueron las que presentaron mayor frecuencia de aumento de peso excesivo.

Conclusión: La prevalencia de aumento de peso gestacional excesivo fue del 38,3 \% y se relacionó con mayores ingresos por cápita, gestación planificada, exceso de peso pregestacional, aumento de la ingesta de alimentos y mayor frecuencia semanal de productos industrializados.

\section{Introdução}

O aumento na prevalência de sobrepeso e obesidade ao longo dos anos, resultou em uma maior proporção de pessoas obesas em todas as faixas etárias, inclusive no início e/ou durante a gravidez. ${ }^{(1)} \mathrm{O}$ aumento do índice de massa corporal (IMC) na gravidez está associado a complicaçóes maternas, como diabetes mellitus, hipertensão gestacional e pré-eclâmpsia, e riscos elevados de macrossomia fetal, defeitos congênitos, mortalidade perinatal e doenças metabólicas em crianças. ${ }^{(2-4)}$ Estas complicações e desfechos são relacionados com a obesidade materna pré-gestacional e o ganho de peso gestacional (GPG) excessivo, sendo esses considerados graves problemas de saúde pública no Brasil e no mundo. ${ }^{(4,5)}$ Portanto, é necessário explorar mais evidências relacionadas ao sobrepeso e obesidade entre mulheres grávidas.

Estudo de revisão com metanálise que incluiu 37 coortes de gravidez e nascimento da Europa, América do Norte e Austrália estimou que de $21,7 \%$ a $41,7 \%$ dos casos de sobrepeso/obesidade na infância foram associados ao sobrepeso e obesidade maternos, enquanto $11,4 \%$ a $19,2 \%$ estiveram relacionados ao ganho de peso gestacional excessivo. ${ }^{(6)}$

No Brasil, anualmente é realizado nas capitais o "Inquérito telefônico nacional de vigilância dos fatores de risco e proteção para doenças crônicas" (VIGITEL), ${ }^{(7)}$ o qual verificou a tendência ascendente nas taxas de excesso de peso e obesidade nas mulheres com idade acima de 18 anos no período de 2006 a 2016. Nesse período, o excesso de peso em adultos aumentou 22\%, alcançando o percen- tual de $50,5 \%$ e a obesidade saltou de $12,1 \%$, para $19,6 \% .{ }^{(8)}$ Considerando o impacto do excesso de peso e obesidade na gravidez/em mulheres grávidas, nos resultados maternos e neonatais, o Ministério da Saúde do Brasil preconiza o monitoramento do peso e avaliação do estado nutricional da gestante em todas as consultas do pré-natal, mediante a verificação de medidas antropométricas, como peso, altura e cálculo do Índice de Massa Corporal (IMC). Trata-se de procedimentos simples, sem custos implicados e que são efetivos para o acompanhamento do estado nutricional, a detecção precoce do ganho ponderal excessivo e o direcionamento das açóes em saúde. ${ }^{(9)}$

No âmbito da Atenção Primária em Saúde (APS), a equipe de enfermagem é responsável pela realização de mensuraçôes durante as pré-consultas. No caso específico do acompanhamento pré-natal, as informaçóes referentes ao peso e altura permitem ao médico e ao enfermeiro responsável pela consulta avaliar a evolução do ganho ponderal e realizar intervençóes específicas para cada caso. Diante disto, é necessário fundamentar as açóes destes profissionais a fim de melhorar a tomada de decisóes frente à identificação de fatores de risco presentes nas gestantes e traçar estratégias de enfrentamento do problema do ganho de peso excessivo nesta população, e assim fortalecer as políticas públicas nesta área da APS. ${ }^{(10)}$

Em contraponto, ainda persistem desafios na APS em relação às açóes de prevenção e controle da obesidade, como a pouca valorização da verificação de medidas antropométricas, entre eles o pouco envolvimento dos profissionais no rastreamento do ganho de peso nas diferentes etapas da vida. Ressalta-se 
que o aumento, ainda que pequeno, da renda e do poder de compra da população brasileira, devido aos programas sociais para famílias de baixa renda, favorece o acesso a produtos alimentícios. Porém, nem sempre esse acesso significa o consumo de alimentos nutritivos e saudáveis. $\mathrm{Na}$ maioria das vezes, as opçóes de baixo custo geralmente são pouco saudáveis, com alto teor de açúcares e carboidratos, cujo consumo aumenta as possibilidades de ganho de peso. ${ }^{(11)}$

Assim, fatores socioculturais, dificuldades no acesso aos serviços de saúde, ${ }^{(12)}$ tanto quanto variáveis obstétricas, comportamentais e socioeconômicas podem se associar ao GPG excessivo. ${ }^{(13)}$ Deste modo, considera-se que a ausência de monitoramento do ganho ponderal e de informaçóes sobre a importância da prática de atividade física e de hábitos alimentares adequados, em especial quanto ao baixo consumo de produtos industrializados, também podem resultar em GPG excessivo. ${ }^{(11)}$

Portanto, a investigação dos preditores do GPG excessivo configura-se como vertente de estudo original e recurso oportuno que pode orientar intervençôes no âmbito das políticas públicas direcionadas às mulheres em idade fértil $\mathrm{e}$, de forma mais específica, durante o período gestacional. Deste modo, este estudo tem como objetivo verificar a prevalência e os fatores associados ao GPG excessivo.

\section{Métodos}

Estudo transversal realizado com uma amostra não probabilística de puérperas residentes no município de Maringá-Paraná, cujo parto foi financiado pelo Sistema Único de Saúde (SUS). Para definir o tamanho amostral de 462 mulheres foi considerado o número aproximado de gestantes do município e prevalência de $13 \%$ para a retenção de peso maior que $5 \mathrm{~kg}$ em mulheres com 12 meses pós-parto. ${ }^{(14)}$

No município de Maringá apenas dois hospitais realizam partos financiados pelo SUS. Assim, o número de puérperas incluídas no estudo foi proporcional ao número de partos ocorridos em cada hospital no ano de 2015.

As participantes do estudo foram localizadas a partir de buscas ativas, realizadas diariamente, nos dois hospitais. Foram abordadas todas as puérperas internadas que atenderam aos seguintes critérios de inclusão - gestação única, de concepto vivo, que pariram com idade gestacional maior ou igual a 37 semanas. Por sua vez, foram excluídas aquelas que não souberam informar ou que não tinham registro do peso pré-gestacional e do peso ao final da gestação no cartão da gestante e mulheres que não compreendiam o idioma português. Todas as participantes realizaram o pré-natal pelo SUS, a maioria delas, integralmente. Contudo, não foi questionado o profissional que realizou as consultas.

Os dados foram coletados no período de dezembro de 2017 a setembro de 2019, durante a internação no pós-parto imediato, mediante entrevista, consulta ao prontuário e cartão da gestante. Foi utilizado um questionário misto, com perguntas fechadas e abertas, elaborado pela própria autora com base nos objetivos do estudo, abordando características antropométricas e gestacionais autorreferidas, aspectos socioeconômicos, história obstétrica, dados da gestação atual e hábitos de vida. Nos prontuários e cartão da gestante foram levantados dados não coletados durante a entrevista, quando, por exemplo, a entrevistada não recordou ou não soube informar. Duas enfermeiras, doutorandas em enfermagem, devidamente treinadas, foram responsáveis pela coleta dos dados.

A variável dependente foi o GPG excessivo, determinado pela diferença entre o peso ao final da gestação e o peso pré-gestacional. Considerou-se como peso pré-gestacional aquele referido até dois meses anterior à concepção ou descoberta da gestação, ou o anotado no cartão do pré-natal até a $14^{\mathrm{a}}$ semana de gestação. Para o peso ao final da gestação considerou-se o citado pela mulher, referente à, no máximo, 30 dias anteriores à data do parto ou o peso anotado no cartão da gestante referente à última consulta pré-natal e que não excedesse 30 dias antes da data do parto.

Para categorização da variável dependente ("sim" ou "não" para GPG excessivo), considerou-se como GPG excessivo quando o ganho foi superior ao recomendado de acordo com o estado nutricional antes da gestação sendo considerado adequado o ganho de peso entre 12,5 e $18 \mathrm{~kg}$ para mulheres com baixo peso; entre 11 e 16 kg para as eutróficas; 
entre 7 e 11,5 kg para as com sobrepeso; e entre 5 e $9 \mathrm{~kg}$ para as obesas. ${ }^{(15)}$

As variáveis independentes foram agrupadas em quatro conjuntos:

a. Socioeconômicas: faixa etária, estado civil, ensino médio (EM) concluído, trabalho remunerado, carteira assinada, raça/cor, bolsa família, renda per capita e classificação econômica (C, D, E) segundo o poder de compra dos indivíduos. ${ }^{(16)}$

b. Obstétricas: multípara, cesariana anterior, parto vaginal anterior, época de início do pré-natal, número de consultas de pré-natal, gestação planejada e tipo de parto atual.

c. Estado nutricional: obesidade familiar materna, desejo de emagrecer, já usou medicamento para emagrecer e estado nutricional inicial segundo IMC classificado em excesso de peso ou normal/baixo peso (BP).

d. Hábitos alimentares: comer rápido, mudança de alimentação na gestaçáo, aumento de ingesta alimentar, aumento do desejo por doces, considera alimentação saudável, recebeu orientação sobre alimentação durante o pré-natal e se condição financeira interfere na alimentação saudável. Para identificar a frequência semanal do consumo de refrigerante/suco artificial adoçado e de produtos industrializados foram utilizadas duas questôes do inquérito VIGITEL. ${ }^{(7)}$

e. Atividade física (AF): trabalho na gestação, esforço físico no trabalho, carro próprio, dirigir frequentemente, sedentarismo na gestação, condição financeira interfere na prática de $\mathrm{AF}$, mudança na $\mathrm{AF}$ durante a gestação, indicação ou proibição de AF por profissional de saúde, AF antes e durante gestação (dividida por trimestres), repouso, atividades diárias e locomoção (divididas nos três trimestres).

A análise descritiva e estatística dos dados foi realizada por meio do software SPSS ${ }^{\oplus}$, sendo realizado teste de associação (Qui-quadrado de Pearson) na análise bivariada, e aquelas que apresentaram valor de $\mathrm{p}<0,20$ foram inseridas no modelo de regressão logística múltipla (método forward). Utilizou-se como medida de associação odds ratio (OR), com intervalo de confiança de $95 \%$. A significância foi estabelecida quando $\mathrm{p}<0,05$ para a manutenção das variáveis no modelo logístico. O nível de ajuste do modelo foi verificado por meio do teste do Hosmer e Lemeshow.

O estudo foi aprovado pelo Comitê de Ética em Pesquisa com Seres Humanos da Universidade Estadual de Maringá (Parecer no 2.180.586). Todas as puérperas que aceitaram participar assinaram o Termo de Consentimento Livre e Esclarecido (TCLE).

\section{Resultados}

A prevalência de GPG excessivo entre as 462 puérperas foi de 38,3\% ( $n=177)$ e foi mais frequente entre as com uniáo estável, trabalho remunerado e com carteira assinada, raça parda/negra/amarela, renda per capita maior que um salário-mínimo, com cesárea anterior, que realizaram sete ou mais consultas, cuja gestação foi planejada e o parto atual foi cesárea (Tabela 1). Na análise bivariada, verificou-se que as variáveis socioeconômicas e obstétricas que puderam ser inseridas na análise múltipla foram: trabalho remunerado $(p=0,06)$, carteira assinada $(p=0,09)$, raça/ cor $(\mathrm{p}=0,19)$, renda per capita $(\mathrm{p}=0,05)$, número de consultas $(p=0,00)$, gestação planejada $(p=0,06)$ e tipo de parto atual $(\mathrm{p}=0,16)$ (Tabela 1).

Em relação ao estado nutricional e hábitos alimentares, o GPG excessivo foi significativamente mais frequente entre as que tinham excesso de peso antes da gestação, que desejavam emagrecer, já usou medicamento para tal, as que aumentaram a ingesta alimentar durante a gestação e que consumiam produtos industrializados três ou mais vezes por semanas. Em relação à atividade física, o GPG excessivo foi significativamente maior entre as que necessitaram de repouso no $1^{\circ}$ trimestre e que não realizaram AF no mesmo trimestre (Tabela 2).

Para análise múltipla foram selecionadas: obesidade familiar materna $(\mathrm{p}=0,15)$, desejo de emagrecer $(\mathrm{p}=0,00)$, estado nutricional inicial $(\mathrm{p}=0,00)$, já usou medicamento para emagrecer $(p=0,05)$, aumento da ingesta alimentar na gestação $(\mathrm{p}=0,00)$, aumento do desejo por doces na gestaçáo $(p=0,13)$, considerar a alimentaçáo saudável $(\mathrm{p}=0,08)$ e a frequência de consumo semanal de produtos industrializados $(\mathrm{p}=0,00)$. Quanto à prática de AF, foram selecionadas: trabalho na gestação $(\mathrm{p}=0,10)$, possuir carro $(\mathrm{p}=0,11)$, seden- 
Tabela 1. Análise bivariada entre ganho de peso excessivo na gestação e características socioeconômicas/obstétricas

\begin{tabular}{|c|c|c|c|c|}
\hline \multirow[b]{2}{*}{ Variáveis } & \multicolumn{4}{|c|}{ Ganho de peso excessivo na gestação } \\
\hline & $\begin{array}{l}\text { Sim } \\
n(\%)\end{array}$ & $\begin{array}{l}\text { Não } \\
\text { n(\%) }\end{array}$ & $\begin{array}{l}\text { Total } \\
\mathrm{n}(\%)\end{array}$ & $p$-value \\
\hline \multicolumn{5}{|l|}{ Faixa etária (em anos) } \\
\hline Menor que 24 & $71(38,0)$ & $116(62,0)$ & $187(40,5)$ & 0,96 \\
\hline 25 ou mais & $105(38,2)$ & $170(61,8)$ & $275(59,5)$ & \\
\hline \multicolumn{5}{|l|}{ Estado civil } \\
\hline Solteira/Viúva/Separada & $18(32,7)$ & $37(67,3)$ & $55(11,9)$ & 0,36 \\
\hline União estável/Casada & $159(39,1)$ & $248(60,9)$ & $407(88,1)$ & \\
\hline \multicolumn{5}{|l|}{ Ens. Médio concluído } \\
\hline Sim & $92(38,2)$ & $149(61,8)$ & $241(52,2)$ & 0,95 \\
\hline Não & $85(38,5)$ & $136(61,5)$ & $221(47,8)$ & \\
\hline \multicolumn{5}{|l|}{ Trabalho remunerado } \\
\hline Sim & $111(42,0)$ & $153(58,0)$ & $264(57,1)$ & 0,06 \\
\hline Não & $66(33,3)$ & $132(66,7)$ & $198(42,9)$ & \\
\hline \multicolumn{5}{|l|}{ Carteira assinada } \\
\hline Sim & $76(43,2)$ & $100(56,8)$ & $176(38,1)$ & 0,09 \\
\hline Não & $101(35,3)$ & $185(64,7)$ & $286(61,9)$ & \\
\hline \multicolumn{5}{|l|}{ Raça/cor } \\
\hline Parda/negra/amarela & $95(41,3)$ & $135(58,7)$ & $230(49,8)$ & 0,19 \\
\hline Branca & $82(35,3)$ & $150(64,7)$ & $232(50,2)$ & \\
\hline \multicolumn{5}{|l|}{ Bolsa família } \\
\hline Sim & $21(31,8)$ & $45(68,2)$ & $66(14,3)$ & 0,24 \\
\hline Não & $156(39,4)$ & $240(60,6)$ & $396(85,7)$ & \\
\hline \multicolumn{5}{|c|}{ Renda per capita (em salário-mínimo*) } \\
\hline$\leq 1$ & $106(35,1)$ & $196(64,9)$ & $302(65,4)$ & 0,05 \\
\hline$>1$ & $71(44,4)$ & $89(55,6)$ & $160(34,6)$ & \\
\hline \multicolumn{5}{|l|}{ Classe econômica C,D,E } \\
\hline Sim & $128(37,4)$ & $214(62,6)$ & $342(74,0)$ & 0,51 \\
\hline Não & $49(40,8)$ & $71(59,2)$ & $120(26,0)$ & \\
\hline \multicolumn{5}{|l|}{ Multípara } \\
\hline Sim & $108(38,3)$ & $174(61,7)$ & $282(61,0)$ & 0,99 \\
\hline Não & $69(38,3)$ & $111(61,7)$ & $180(39,0)$ & \\
\hline \multicolumn{5}{|l|}{ Cesariana anterior ${ }^{\star \star}$} \\
\hline Sim & $69(40,8)$ & $100(59,2)$ & $169(59,9)$ & 0,29 \\
\hline Não & $39(34,5)$ & $74(65,5)$ & $113(40,1)$ & \\
\hline \multicolumn{5}{|l|}{ Parto vaginal anterior** } \\
\hline Sim & $41(35,7)$ & $74(64,3)$ & $115(40,8)$ & 0,45 \\
\hline Não & $67(40,1)$ & $100(59,9)$ & $167(59,2)$ & \\
\hline \multicolumn{5}{|l|}{ Início pré-natal } \\
\hline $\mathrm{IG}<12$ sem & $137(39,5)$ & $210(60,5)$ & $347(76,1)$ & 0,39 \\
\hline$I G \geq 12$ sem & $38(34,9)$ & $71(65,1)$ & $109(23,9)$ & \\
\hline \multicolumn{5}{|l|}{$\mathrm{N}^{0}$ de consultas ${ }^{\star \star \star}$} \\
\hline$\geq 7$ & $156(42,2)$ & $214(57,8)$ & $370(81,3)$ & 0,00 \\
\hline$\leq 6$ & $19(22,4)$ & $66(77,6)$ & $85(18,7)$ & \\
\hline \multicolumn{5}{|l|}{ Gestação planejada ${ }^{\star \star \star \star}$} \\
\hline Sim & $63(44,7)$ & $78(55,3)$ & $141(30,7)$ & 0,06 \\
\hline Não & $113(35,5)$ & $205(64,5)$ & $318(69,3)$ & \\
\hline \multicolumn{5}{|l|}{ Parto atual } \\
\hline Vaginal & $67(34,5)$ & $127(65,6)$ & $194(42,0)$ & 0,16 \\
\hline Cesariana & $110(41,0)$ & $158(59,0)$ & $268(58,0)$ & \\
\hline
\end{tabular}

${ }^{*}$ Qui-quadrado; ${ }^{\star \star} \mathrm{n}=282 ;{ }^{* \star \star} \mathrm{n}=455$; ${ }^{\star \star \star \star} \mathrm{n}=459$; "Salário-mínimo referente ao ano de 2018, cotado no valor de $\mathrm{R} \$ 965,00$

tarismo na gestação $(\mathrm{p}=0,17)$, se condição financeira interfere na prática de atividade física $(\mathrm{p}=0,15)$, repouso no $1^{\circ}$ e $2^{\circ}$ trimestre $(p=0,01 ; p=0,08)$, AF no $1^{\circ}$ trimestre $(\mathrm{p}=0,06)$, AF como meio de transporte no $1^{\circ}$, $2^{\circ}$ e $3^{\circ}$ trimestre $(\mathrm{p}=0,17 ; \mathrm{p}=0,14 ; \mathrm{p}=0,10)$ (Tabela 2$)$.
Tabela 2. Análise bivariada entre ganho de peso excessivo na gestação, estado nutricional, hábitos alimentares e prática de atividade física

\begin{tabular}{|c|c|c|c|c|}
\hline \multirow[b]{2}{*}{ Variáveis } & \multicolumn{4}{|c|}{ Ganho de peso excessivo na gestação } \\
\hline & $\begin{array}{l}\text { Sim } \\
n(\%)\end{array}$ & $\begin{array}{l}\text { Não } \\
\text { n(\%) }\end{array}$ & $\begin{array}{l}\text { Total } \\
\text { n(\%) }\end{array}$ & $p$-value \\
\hline \multicolumn{5}{|c|}{ Obesidade familiar materna } \\
\hline Sim & $36(45,6)$ & $43(54,4)$ & $79(17,1)$ & 0,15 \\
\hline Não & $141(36,8)$ & $242(63,2)$ & $383(82,9)$ & \\
\hline \multicolumn{5}{|l|}{ Desejo de emagrecer } \\
\hline Sim & $105(47,7)$ & $115(52,3)$ & $220(47,6)$ & 0,00 \\
\hline Não & $72(29,8)$ & $170(70,2)$ & $242(52,4)$ & \\
\hline \multicolumn{5}{|c|}{ Estado nutricional inicial } \\
\hline Excesso de peso & $118(49,6)$ & $120(50,4)$ & $238(51,5)$ & 0,00 \\
\hline Normal/BP & $59(26,3)$ & $165(73,7)$ & $224(48,5)$ & \\
\hline \multicolumn{5}{|l|}{ Comer rápido } \\
\hline $\operatorname{Sim}$ & $60(42,3)$ & $82(57,7)$ & $142(69,3)$ & 0,25 \\
\hline Não & $117(36,6)$ & $203(63,4)$ & $320(30,7)$ & \\
\hline \multicolumn{5}{|c|}{ Já usou medicamento emagrecer } \\
\hline $\operatorname{Sim}$ & $29(50,0)$ & $29(50,0)$ & $58(12,6)$ & 0,05 \\
\hline Não & $148(36,6)$ & $256(63,4)$ & $404(87,4)$ & \\
\hline \multicolumn{5}{|c|}{ Mudança alimentação na gestação } \\
\hline Sim & $100(37,7)$ & $165(62,3)$ & $265(57,4)$ & 0,77 \\
\hline Não & $77(39,1)$ & $120(60,9)$ & $197(42,6)$ & \\
\hline \multicolumn{5}{|c|}{ Aumento ingesta alimentar na gestação } \\
\hline Sim & $91(49,2)$ & $94(50,8)$ & $185(40,0)$ & 0,00 \\
\hline Não & $86(31,0)$ & $191(69,0)$ & $277(60,0)$ & \\
\hline \multicolumn{5}{|c|}{ Aumento desejo doces na gestação } \\
\hline Sim & $121(40,9)$ & $175(59,1)$ & $296(64,1)$ & 0,13 \\
\hline Não & $56(33,7)$ & $110(66,3)$ & $166(35,9)$ & \\
\hline \multicolumn{5}{|c|}{ Considera alimentação saudável } \\
\hline $\operatorname{Sim}$ & $84(34,6)$ & $159(65,4)$ & $243(52,6)$ & 0,08 \\
\hline Não & $93(42,5)$ & $126(57,5)$ & $219(47,4)$ & \\
\hline \multicolumn{5}{|c|}{$\begin{array}{l}\text { Recebeu orientação sobre alimentação } \\
\text { durante o pré-natal }\end{array}$} \\
\hline Sim & $103(36,4)$ & $180(63,6)$ & $283(61,3)$ & 0,29 \\
\hline Não & $74(41,3)$ & $105(58,7)$ & $179(38,7)$ & \\
\hline \multicolumn{5}{|c|}{$\begin{array}{l}\text { Condição financeira interfere alimentação } \\
\text { saudável }\end{array}$} \\
\hline Sim & $62(37,8)$ & $102(62,2)$ & $164(35,5)$ & 0,87 \\
\hline Não & $115(38,6)$ & $183(61,4)$ & $298(64,5)$ & \\
\hline \multicolumn{5}{|c|}{$\begin{array}{l}\text { Frequência semanal consumo refrigerante/ } \\
\text { suco artificial adoçado }\end{array}$} \\
\hline 3 vezes ou mais & $93(40,4)$ & $137(59,6)$ & $230(49,8)$ & 0,35 \\
\hline 2 vezes ou menos & $84(36,2)$ & $148(63,8)$ & $232(50,2)$ & \\
\hline \multicolumn{5}{|c|}{$\begin{array}{l}\text { Frequência semanal consumo } \\
\text { industrializados }\end{array}$} \\
\hline 3 vezes ou mais & $114(45,6)$ & $136(54,4)$ & $250(54,1)$ & 0,00 \\
\hline 2 vezes ou menos & $63(29,7)$ & $149(70,3)$ & $212(45,9)$ & \\
\hline \multicolumn{5}{|l|}{ Trabalhou gestação } \\
\hline Sim & $98(42,1)$ & $135(57,9)$ & $233(50,4)$ & 0,10 \\
\hline Não & $79(34,5)$ & $150(65,5)$ & $229(49,6)$ & \\
\hline \multicolumn{5}{|c|}{ Esforço físico no trabalho** } \\
\hline Sim & $30(39,5)$ & $46(60,5)$ & $76(32,6)$ & 0,58 \\
\hline Não & $68(43,3)$ & $89(56,7)$ & $157(67,4)$ & \\
\hline \multicolumn{5}{|l|}{ Possuir carro } \\
\hline Sim & $124(40,9)$ & $179(59,1)$ & $303(65,6)$ & 0,11 \\
\hline Não & $53(33,3)$ & $106(66,7)$ & $159(34,4)$ & \\
\hline \multicolumn{5}{|c|}{ Dirige frequentemente } \\
\hline Sim & $77(39,3)$ & $119(60,7)$ & $196(42,4)$ & 0,71 \\
\hline Não & $100(37,6)$ & $166(62,4)$ & $266(57,6)$ & \\
\hline
\end{tabular}




\begin{tabular}{|c|c|c|c|c|}
\hline \multirow[b]{2}{*}{ Variáveis } & \multicolumn{4}{|c|}{ Ganho de peso excessivo na gestação } \\
\hline & $\begin{array}{l}\text { Sim } \\
\text { n(\%) }\end{array}$ & $\begin{array}{l}\text { Não } \\
\text { n(\%) }\end{array}$ & $\begin{array}{l}\text { Total } \\
\mathrm{n}(\%)\end{array}$ & $p$-value \\
\hline \multicolumn{5}{|c|}{ Sedentarismo na gestação } \\
\hline $\operatorname{Sim}$ & $141(40,1)$ & $211(59,9)$ & $352(76,2)$ & 0,17 \\
\hline Não & $36(32,7)$ & $74(67,3)$ & $110(23,8)$ & \\
\hline \multicolumn{5}{|c|}{ Condição financeira interfere prática AF } \\
\hline $\operatorname{Sim}$ & $49(44,1)$ & $62(55,9)$ & $111(24,0)$ & 0,15 \\
\hline Não & $128(36,5)$ & $223(63,5)$ & $351(76,0)$ & \\
\hline \multicolumn{5}{|l|}{ AF antes gestação } \\
\hline Sim & $52(40,6)$ & $76(59,4)$ & $128(27,7)$ & 0,53 \\
\hline Não & $125(37,4)$ & $209(62,6)$ & $334(72,3)$ & \\
\hline \multicolumn{5}{|c|}{ Mudança na AF durante a gestação } \\
\hline $\operatorname{Sim}$ & $62(42,2)$ & $85(57,8)$ & $147(31,8)$ & 0,24 \\
\hline Não & $115(36,5)$ & $200(63,5)$ & $315(68,2)$ & \\
\hline \multicolumn{5}{|c|}{ Indicação de AF por profissional saúde } \\
\hline Sim & $96(40,5)$ & $141(59,5)$ & $237(51,3)$ & 0,32 \\
\hline Não & $81(36,0)$ & $144(64,0)$ & $225(48,7)$ & \\
\hline \multicolumn{5}{|c|}{ Proibição AF por profissional saúde } \\
\hline Sim & $21(46,7)$ & $24(53,3)$ & $45(9,7)$ & 0,23 \\
\hline Não & $156(37,4)$ & $261(62,6)$ & $417(90,3)$ & \\
\hline \multicolumn{5}{|l|}{ Repouso $1^{\circ}$ trimestre } \\
\hline $\operatorname{Sim}$ & $30(53,6)$ & $26(46,4)$ & $56(12,1)$ & 0,01 \\
\hline Não & $147(36,2)$ & $259(63,8)$ & $406(87,9)$ & \\
\hline \multicolumn{5}{|l|}{ Repouso $2^{\circ}$ trimestre } \\
\hline $\operatorname{Sim}$ & $24(50,0)$ & $24(50,0)$ & $48(10,4)$ & 0,08 \\
\hline Não & $153(37,0)$ & $261(63,0)$ & $414(89,6)$ & \\
\hline \multicolumn{5}{|l|}{ Repouso $3^{\circ}$ trimestre } \\
\hline Sim & $41(41,4)$ & $58(58,6)$ & $99(21,4)$ & 0,48 \\
\hline Não & $136(37,5)$ & $227(62,5)$ & $363(78,6)$ & \\
\hline \multicolumn{5}{|l|}{ AF $1^{\circ}$ trimestre } \\
\hline $\operatorname{Sim}$ & $18(27,7)$ & $47(72,3)$ & $65(14,1)$ & 0,06 \\
\hline Não & $159(40,1)$ & $238(59,9)$ & $397(85,9)$ & \\
\hline \multicolumn{5}{|l|}{ AF $2^{\circ}$ trimestre } \\
\hline Sim & 22(33,8) & $43(66,2)$ & $65(14,1)$ & 0,42 \\
\hline Não & $155(39,0)$ & $242(61,0)$ & $397(85,9)$ & \\
\hline \multicolumn{5}{|l|}{$\mathrm{AF} 3^{\circ}$ trimestre } \\
\hline $\operatorname{Sim}$ & $19(35,8)$ & $34(64,2)$ & $53(11,5)$ & 0,70 \\
\hline Não & $158(38,6)$ & $251(61,4)$ & $409(88,5)$ & \\
\hline \multicolumn{5}{|l|}{$A D 1^{\circ}$ trimestre } \\
\hline $\operatorname{Sim}$ & $117(36,7)$ & $202(63,3)$ & $319(69,0)$ & 0,28 \\
\hline Não & $60(42,0)$ & $83(58,0)$ & $143(31,0)$ & \\
\hline \multicolumn{5}{|l|}{$\mathrm{AD} 2^{\circ}$ trimestre } \\
\hline $\operatorname{Sim}$ & $109(36,6)$ & $189(63,4)$ & $298(64,5)$ & 0,30 \\
\hline Não & $68(41,5)$ & $96(58,5)$ & $164(35,5)$ & \\
\hline \multicolumn{5}{|l|}{$\mathrm{AD} 3^{\circ}$ trimestre } \\
\hline $\operatorname{Sim}$ & $95(36,8)$ & $163(63,2)$ & $258(55,8)$ & 0,46 \\
\hline Não & $82(40,2)$ & $122(59,8)$ & $204(44,2)$ & \\
\hline \multicolumn{5}{|c|}{ AF como Meio transporte $1^{\circ}$ trimestre } \\
\hline Nenhum & $106(41,1)$ & $152(58,9)$ & $258(55,8)$ & 0,17 \\
\hline Caminhada/bicicleta & $71(34,8)$ & $133(65,2)$ & $204(44,2)$ & \\
\hline \multicolumn{5}{|c|}{ AF como Meio transporte $2^{\circ}$ trimestre } \\
\hline Nenhum & $110(41,2)$ & $157(58,8)$ & $267(57,8)$ & 0,14 \\
\hline Caminhada/bicicleta & $67(34,4)$ & $128(65,6)$ & $195(42,2)$ & \\
\hline AF como Meio transpor & & & & \\
\hline Nenhum & $118(41,3)$ & $168(58,7)$ & $286(61,9)$ & 0,10 \\
\hline Caminhada/bicicleta & $59(33,5)$ & $117(66,5)$ & $176(38,1)$ & \\
\hline
\end{tabular}

${ }^{*}$ Qui-quadrado; ${ }^{* *} \mathrm{n}=233$
Na tabela 3 é apresentado o resultado da análise múltipla indicando que cinco variáveis permaneceram no modelo final e constituem os fatores associados ao GPG excessivo. Observou-se menor frequência de GPG excessivo com renda per capita menor/ igual a um salário-mínimo, resultando em menor chance $(\mathrm{OR}=0,603)$ por parte destas mulheres de apresentarem o desfecho negativo.

As gestantes que referiram ter planejado a gestação $(\mathrm{OR}=1,562)$, tinham excesso de peso pré-gestacional $(\mathrm{OR}=3,252)$, aumentaram a ingesta alimentar na gestação $(\mathrm{OR}=2,496)$ e com frequência de consumo semanal de produtos industrializados de três vezes ou mais $(\mathrm{OR}=1,949)$ foram aquelas com maior chance relativa de apresentarem o GPG excessivo (Tabela 3).

Tabela 3. Fatores associados ao ganho de peso excessivo na gestação a partir da análise de regressão logística

\begin{tabular}{|c|c|c|c|c|}
\hline \multirow[b]{2}{*}{ Variáveis } & \multicolumn{4}{|c|}{ Ganho de peso excessivo na gestação } \\
\hline & $\begin{array}{l}\text { Sim } \\
n(\%)\end{array}$ & $\begin{array}{l}\text { Não } \\
n(\%)\end{array}$ & OR (IC95\%) & $\mathrm{p}$-value \\
\hline \multicolumn{5}{|l|}{ Renda per capita } \\
\hline$\leq 1 \mathrm{~s} \cdot \mathrm{m}^{*}$ & $106(35,1)$ & $196(64,9)$ & \multirow{2}{*}{$\begin{array}{c}\mathrm{OR}=0,6031 \\
(0,3941-0,925)\end{array}$} & \multirow[t]{2}{*}{0,020} \\
\hline$>1 \mathrm{~s} . \mathrm{m}$ & $71(44,4)$ & $89(55,6)$ & & \\
\hline \multicolumn{5}{|l|}{ Gestação planejada } \\
\hline Sim & $63(44,7)$ & $78(55,3)$ & \multirow{2}{*}{$\begin{array}{c}\mathrm{OR}=1,562 \\
(1,005-2,427)\end{array}$} & \multirow{2}{*}{0,048} \\
\hline Não & $113(35,5)$ & $205(64,5)$ & & \\
\hline \multicolumn{5}{|l|}{ Estado nutricional inicial } \\
\hline Excesso de peso & $118(49,6)$ & $120(50,4)$ & \multirow{2}{*}{$\begin{array}{c}\mathrm{OR}=3,252 \\
(2,132-4,962)\end{array}$} & \multirow{2}{*}{$<0,001$} \\
\hline Normal/BP & $59(26,3)$ & $165(73,7)$ & & \\
\hline \multicolumn{5}{|l|}{$\begin{array}{l}\text { Aumento da ingesta } \\
\text { alimentar na gestação }\end{array}$} \\
\hline $\operatorname{Sim}$ & $91(49,2)$ & $94(50,8)$ & \multirow{2}{*}{$\begin{array}{c}\mathrm{OR}=2,496 \\
(1,634-3,812)\end{array}$} & \multirow{2}{*}{$<0,001$} \\
\hline Não & $86(31,0)$ & $191(69,0)$ & & \\
\hline \multicolumn{5}{|c|}{$\begin{array}{l}\text { Frequência semanal do } \\
\text { consumo de industrializados }\end{array}$} \\
\hline 3 vezes ou mais & $114(45,6)$ & $136(54,4)$ & \multirow{2}{*}{$\begin{array}{c}\mathrm{OR}=1,949 \\
(1,283-2,961)\end{array}$} & \multirow{2}{*}{0,002} \\
\hline 2 vezes ou menos & $63(29,7)$ & $149(70,3)$ & & \\
\hline
\end{tabular}

*Salário-mínimo referente ao ano de 2018, cotado no valor de $\mathrm{R} \$ 965,00$; ** 0 btido em análise múltipla de regressão logística

\section{Discussão}

Destacam-se como possíveis limitaçóes do estudo, a ausência/deficiência de registro relacionado ao peso pré e pós-gestacional no cartão da gestante e no prontuário, o que não permitiu que uma única estratégia para levantamento desta informação fosse adotada; assim como a não identificação de possíveis variáveis de confusão na definição do método.

De qualquer modo, os resultados encontrados podem subsidiar discussóes entre gestores e profis- 
sionais de saúde, em especial os enfermeiros que por vezes atuam como líderes de equipe, quanto à importância da realização de intervençóes educativas com foco não só nos fatores de risco para doenças crônicas não transmissíveis, mas também aqueles que podem comprometer a saúde da mulher e de seu concepto durante a gravidez, parto e puerpério.

Neste sentido, ênfase especial deve ser direcionada às mulheres em idade fértil, pois foi identificado que o estado nutricional pré-gestacional constitui fator associado ao GPG excessivo. Do mesmo modo, devem ser implementadas açóes de monitoramento, com o estabelecimento de indicadores e metas a serem alcançados junto às gestantes residentes no território de abrangência de cada Unidade Básica de Saúde/Equipe da Estratégia Saúde da Família.

A prevalência de 38,3\% de GPG excessivo entre as puérperas foi um pouco inferior à encontrada em estudo chinês com 1.643 mulheres $(43,2 \%) .{ }^{(17)}$ No entanto, esse achado reitera a importância do rastreio e monitoramento do ganho de peso ao longo de toda gestação, a fim de possibilitar intervençóes precoces no combate a complicaçóes tanto à gestante quanto à criança.

No que concerne à renda, apesar de grande parte das participantes pertencerem às classes $\mathrm{C}, \mathrm{D}$ e $\mathrm{E}$, a frequência de GPG excessivo foi maior entre aquelas com renda per capita maior do que um salário-mínimo. Vale considerar que estas mulheres podem ter mais acesso e consumo de alimentos, sobretudo os processados e industrializados, o que aumenta o risco de GPG excessivo. Contudo, este resultado diverge daqueles observados na literatura, pois no nordeste brasileiro foi identificada associação de GPG excessivo e renda familiar menor que um salário-mínimo, ${ }^{(12)}$ enquanto estudo realizado no interior paulista, identificou associação entre renda per capita maior e GPG adequado. ${ }^{(18)}$

Na população estudada planejar a gestação apresentou relação significativa com o GPG excessivo, divergindo de estudo realizado em município do nordeste brasileiro. ${ }^{(19)}$ Uma vez que a gravidez não planejada pode predispor problemas para a vida da mulher, sobretudo, aquelas em situação de vulnerabilidade socioeconômica ou nutricional, ressalta-se a relevância de aconselhamento antes da concepção, de mulheres obesas ou com sobrepeso que desejam engravidar. ${ }^{(20)}$

Cabe destacar que, iniciar a gestação com o IMC elevado ou ter IMC elevado no primeiro trimestre favorece o ganho excessivo de peso ao longo da gestação, e se associa à ocorrência de sintomas depressivos. ${ }^{(21)}$ Estudo realizado com 607 puérperas, em Maringá Paraná, identificou que IMC pré-gestacional elevado constitui um dos fatores associados ao alto risco gestacional. ${ }^{(22)}$

Isto é preocupante, pois segundo a Organização Mundial de Saúde (OMS), de 2005 a 2013, na América Latina e no Caribe, mais de $50 \%$ das mulheres com idade entre 15 e 49 anos, residentes em áreas urbanas, estavam com sobrepeso ou obesas. ${ }^{(23)}$ No Brasil, não é diferente, pois em 2016 a pesquisa Vigitel identificou que $50,5 \%$ das mulheres acima de 18 anos tinham excesso de peso e $19,6 \%$ obesidade. ${ }^{(7)}$

Evidencia-se que a amostra estudada foi constituída por mulheres com alta prevalência de excesso de peso pré-gestacional e que parcela considerável delas, teve aumento excessivo de peso na gestação. Este resultado corrobora os de pesquisa realizada nos Estados Unidos, que no período de 2000 a 2009, observou crescimento no percentual de mulheres com GPG excessivo, o que esteve associado com sobrepeso e obesidade antes da gestação. ${ }^{(24)}$ e também resultados de uma coorte prospectiva realizada no Canadá, que apontou o excesso de peso pré-gestacional como um dos fatores que favorece o GPG excessivo. ${ }^{(25)}$

Este aspecto precisa ser considerado pelos profissionais de saúde, pois o IMC elevado associa-se à ocorrência de complicaçóes durante a gravidez e parto, além de diabetes gestacional, distúrbios hipertensivos, macrossomia, laceraçóes perineais e de trajeto, aumentando o risco de sangramento pós-parto. Em decorrência dessas associaçóes, o IMC materno tem sido utilizado como preditor da via de parto, aumentando o risco de cesariana quando maior que $30 \mathrm{~kg} / \mathrm{m}^{2}$. ${ }^{(20)}$ Inclusive, estudo realizado a partir de registros administrativos de 216.481 nascimentos ocorridos em Nova Iorque apontou que o excesso de peso e a obesidade pré-gestacionais contribuem proporcionalmente para o maior risco de 
cesárea entre mulheres negras e hispânicas, quando comparadas as mulheres brancas ou asiáticas. ${ }^{(26)}$

Dentre os fatores que contribuem para o IMC alterado está o sedentarismo, o qual apresentou alta proporçáo entre as participantes do estudo. Este fato pode estar relacionado à falta de segurança dos profissionais de saúde em recomendar a prática de atividade física durante a gestação, preocupados com a restrição de crescimento intrauterino, abortamento, lesão musculoesquelética, ou parto prematuro. Estes aspectos, no entanto, não têm sido comprovados em gestações sem complicaçôes. ${ }^{(27)}$

Ademais, cabe ressaltar que os hábitos alimentares também influenciam no ganho de peso durante a gestação. Desse modo, os profissionais de saúde devem ter conhecimento e em sua atuaçáo, valorizarem a forte relação existente entre padrão alimentar baseado em fast foods, alimentos processados, lanches e açucares e o GPG. ${ }^{(28)}$ Destarte, o maior consumo destes alimentos prejudica a saúde materno-fetal por aumentar apenas o ganho energético, predispor a hiperglicemia, porém sem aporte nutricional adequado. ${ }^{(29)}$

Salienta-se que o uso de alimentos industrializados por gestantes tem sido relatado, sobretudo, devido à maior adesão a hábitos alimentares considerados mais rápidos, do tipo fast food. Esta variável deve ser considerada na avaliação dos hábitos alimentares, pois já foram identificadas alteraçôes metabólicas como hiperglicemia e pressão arterial elevada associadas à insegurança alimentar entre gestantes atendidas na rede pública. ${ }^{(29)}$

Estudo realizado no interior do sudeste brasileiro, por exemplo, constatou baixa frequência no consumo de alimentos industrializados entre gestantes, o que foi atribuído ao fato de elas terem realizado acompanhamento nutricional. ${ }^{(30)}$ Contudo, em nosso país, a maioria das gestantes não tem acesso a um nutricionista, por isso é necessário que outros profissionais que assistem as mulheres durante a gestação tenham conhecimento sobre estes aspectos para atendê-las de forma integral. ${ }^{(31)}$

Considerando que o peso constitui um fator modificável e que as mulheres apresentam maior disposição para seguir orientaçôes de saúde durante o período gestacional, destaca-se a necessidade de ser dada maior ênfase a esta temática nas consultas de pré-natal e nos grupos de gestantes. Neste sentido, a equipe multiprofissional, em especial os enfermeiros, devem reforçar a importância, tanto para a mãe quanto para o bebê, de uma dieta saudável, variada e equilibrada.

Vale destacar que no âmbito da Atenção Primária, os enfermeiros têm importante papel no planejamento e na assistência às mulheres durante o ciclo gravídico-puerperal, incluindo o cuidado e acompanhamento da mulher e seu filho desde o período gestacional e puerperal. ${ }^{(9)}$ Estudo aponta que o tipo de relação e o vínculo estabelecido com o enfermeiro, especialmente nos contatos iniciais pode influenciar o modo como as mulheres vão se comportar em relaçáo às orientaçóes de saúde ao longo de toda a gestação. Entretanto, apesar do Ministério da Saúde recomendar que nas gestaçôes de médio e baixo risco as consultas sejam intercaladas entre médico e enfermeiro, nem sempre essa rotina é garantida em todas as unidades, seja por dimensionamento inadequado de pessoal ou de infraestrutura de trabalho. A atuação do enfermeiro no pré-natal ainda é limitada por fatores diversos como o fato de a assistência ainda ser baseada quase que exclusivamente no modelo biomédico, e a ausência de reconhecimento e mesmo desconhecimento do trabalho do enfermeiro por parte da população e de outros profissionais de saúde. No que concerne à própria categoria pode-se citar a necessidade de qualificaçáo profissional, a falta de protocolos e de sistematização na realização de consultas (muitas vezes implementadas para suprir a ausência do profissional médico), deficiência de estrutura física e acúmulo de função. ${ }^{(32,33)}$

A gestação é um período de contatos frequentes com o serviço de saúde. Prova disto é que a maioria das mulheres em estudo realizou sete ou mais consultas, condição propícia para a adequada vigilância do estado nutricional e oferta de informaçôes de qualidade. $\mathrm{O}$ enfermeiro deve ter potencial para acolher, assistir e incentivar hábitos saudáveis durante e após o período gestacional ${ }^{(32)}$ e com vistas à prevenção do GPG excessivo, favorecer o bem-estar físico, mental e a autoestima, e desmistificar mitos em relação à gestação. 
Pondera-se que a construção e discussão de planos alimentares adequados juntos às gestantes, que considerem na medida do possível, as preferências alimentares, as características de contexto familiar e as condiçóes de acesso a alimentos saudáveis, aumentam as chances de eles serem seguidos.

Nesta direção, estudo de intervenção com gestantes obesas e com sobrepeso, constatou que o grupo que teve orientação de uma dieta com menor consumo de açúcar e gordura saturada mostrou menor risco de apresentar complicaçóes. ${ }^{(34)}$ Por sua vez, estudo de revisão com ensaios controlados, randomizados de intervenção dietética e de exercícios, concluiu que dieta ou exercício, ou ambos, durante a gravidez pode reduzir o risco de GPG excessivo. Outros benefícios incluem menor risco de parto por cesariana, ocorrência de hipertensão materna, macrossomia e morbidade respiratória neonatal. ${ }^{(35)}$ Destarte, intervençóes abrangentes no estilo de vida com foco na redução da ingestão calórica e na adoção de uma nutriçáo equilibrada podem efetivamente reduzir o excesso de ganho de peso gestacional. ${ }^{(26)}$

Ainda, reitera-se a importância da enfermagem no cuidado durante o pré-natal, parto e pós-parto, sendo corresponsável pela promoção em saúde, juntamente com a equipe multiprofissional. (10) Ressalta-se que por intermédio das atividades educativas realizadas durante o pré-natal, é possível empoderar as mulheres para o cuidado de si e do bebê. ${ }^{(36,37)}$ Referente a isso, torna-se necessária à abordagem dos fatores de risco ao excesso de peso, em especiais os modificáveis, em todos os encontros/interaçóes com as gestantes, sejam eles realizados durante a consulta de enfermagem ou nas atividades desenvolvidas em grupo.

Por fim, reforça-se que aspectos econômicos e o planejamento da gestação também podem predizer o GPG excessivo, para além dos fatores nutricionais. Em termos práticos, estes fatores destacam a pertinência de açôes das equipes da Atenção Primária à Saúde, em articulação com demais serviços que ofertam o acompanhamento pré-natal, de modo a intervir na identificação e minimização de riscos. Conhecer os fatores relacionados ao ganho de peso gestacional é importante para o enfermeiro conduzir melhor suas ações durante a consulta pré-natal, prevenir complicaçóes obstétricas e neonatais, promover a saúde cardiovascular da gestante refletindo assim na construção de melhores indicadores de saúde com vistas ao alcance das metas estabelecidas pelos Objetivos do Desenvolvimento Sustentável Agenda 2030 das Naçóes Unidas.

\section{Conclusão}

A prevalência de ganho de peso gestacional excessivo foi de $38,3 \%$ e os fatores associados foram: maior renda per capita, gestação planejada, excesso de peso pré-gestacional, aumento da ingesta alimentar e maior frequência semanal no consumo de produtos industrializados.

\section{Colaborações}

Monteschio LVC, Marcon SS, Arruda GO, Teston EF, Nass EMA, Costa JR, Oriá MOB e Pereira ALF contribuíram com a concepção do estudo, análise e interpretação dos dados, redação do artigo, revisão crítica relevante do conteúdo intelectual e aprovaçáo da versão final a ser publicada.

\section{Referências}

1. Flannery C, Mtshede MN, McHugh S, Anaba AE, Clifford E, O'Riordan M, et al. Dietary behaviours and weight management: $A$ thematic analysis of pregnant women's perceptions. Matern Child Nutr. 2020;16(4):e13011.

2. Poston L, Caleyachetty R, Cnattingius S, Corvalán C, Uauy R, Herring S, et al. Preconceptional and maternal obesity: epidemiology and health consequences. Lancet Diabetes Endocrinol. 2016;4(12):1025-1036. Review.

3. Liu P, Xu L, Wang Y, Zhang Y, Du Y, Sun Y, et al. Association between perinatal outcomes and maternal pre-pregnancy body mass index. Obes Rev. 2016;17(11):1091-102. Review.

4. Chen $C, X u X$, Yan $Y$. Estimated global overweight and obesity burden in pregnant women based on panel data model. PLOS One. 2018;13(8):e0202183.

5. Sogunle E, Masukume G, Nelson G. The association between caesarean section delivery and later life obesity in 21-24 year olds in an Urban South African birth cohort. PLoS One. 2019;14(11):e0221379.

6. Voerman E, Santos S, Patro Golab B, Amiano P, Ballester F, Barros H, et al. Maternal body mass index, gestational weight gain, and the risk of overweight and obesity across childhood: an individual participant data meta-analysis. PLoS Med. 2019;16(2):e1002744. 
7. Brasil. Ministério da Saúde. Secretaria de Vigilância em Saúde. Departamento de Vigilância de Doenças e Agravos não Transmissíveis e Promoção da Saúde. Vigitel Brasil 2017. Vigilância de fatores de risco e proteção para doenças crônicas por inquérito telefônico. Brasília (DF): Ministério da Saúde; 2017 [citado 2021 Mar 2021]. Disponível em: https://bvsms.saude.gov.br/bvs/publicacoes/vigitel_brasil_2017_ vigilancia_fatores_riscos.pdf

8. Araújo FG, Velasquez-Melendez G, Felisbino-Mendes MS. Prevalence trends of overweight, obesity, diabetes and hypertension among Brazilian women of reproductive age based on sociodemographic characteristics. Health Care Women Int. 2019;40(4):386-406.

9. Brasil. Ministério da Saúde. Secretaria de Atenção à Saúde. Departamento de Atenção Básica. Atenção ao pré- natal de baixo risco. Brasília (DF): Ministério da Saúde; 2012 [citado 2021 Mar 6]. Disponível em: http://bvsms.saude.gov.br/bvs/publicacoes/atencao_ pre_natal_baixo_risco.pdf

10. Correa MS, Feliciano KV, Pedrosa EN, Souza Al. Acolhimento no cuidado à saúde da mulher no puerpério. Cad Saude Publica. 2017;33(3):e00136215.

11. Almeida LM, Campos KF, Randow R, Guerra VA. Estratégias e desafios da gestão da Atenção Primária à Saúde no controle e prevenção da obesidade Rev Gestão Saúde. 2017;8(1):114-39.

12. Magalhães EI, Maia DS, Bonfim CF, Netto MP, Lamounier JA, Rocha DS. Prevalence and factors associated with excessive weight gain in pregnancy in health units in the southwest of Bahia. Rev Bras Epidemiol. 2015;18(4):858-69.

13. Petursdottir Maack $H$, Skalkidou A, Sjöholm A, Eurenius-Orre $K$, Mulic-Lutvica A, Wikström AK, et al. Maternal body mass index moderates antenatal depression effects on infant birthweight. Sci Rep. 2019;9(6213).

14. Rode L, Kjærgaard H, Ottensen B, Damm P, Hegaard HK. Association between gestational weight gain according to bodymass index and postpartum weight in a large cohort of danish women. Matern Child Health J. 2012;16(2):406-13.

15. Institute of Medicine (US) and National Research Council (US) Committee to Reexamine IOM Pregnancy Weight Guidelines; Rasmussen KM, Yaktine AL, editors. Weight Gain During Pregnancy: Reexamining the Guidelines. Washington (DC): National Academies Press (US); 2009. Summary. Available from: https://www.ncbi.nlm.nih. gov/books/NBK32799/

16. Associação Brasileira de Empresas e Pesquisas (ABEP). Critério de classificação econômica. Critério Brasil 2015 e atualização da distribuição de classes para 2016. São Paulo: ABEP; 2016 [citado 2017 Dez 05]. Disponível em: http://www.abep.org/criterio-brasil

17. Ma D, Szeto IM, Yu K, Ning Y, Li W, Wang J, et al. Association between gestational weight gain according to prepregnancy body mass index and short postpartum weight retention in postpartum women. Clin Nutr. 2015;34(2):291-5

18. Leite T, Port AC. Fatores associados ao ganho ponderal de gestantes atendidas na Unidade básica de saúde da mulher "Maria de Lourdes Campos Silva" em Monte Azul Paulista - SP. Rev Ciênc Nutr Online. 2018;2(2):26-31.

19. Silva LO, Alexandre, MR, Cavalcante AC, Arruda SP, Sampaio R Adequate versus inadequate weight gain and socioeconomic factors of pregnant women followed up in primary care. Rev Bras Saúde Mater Infant. 2019;19(1):107-14.

20. Pettersen-Dahl A, Murzakanova G, Sandvik L, Laine K. Maternal body mass index as a predictor for delivery method. Acta Obstet Gynecol Scand. 2018;97(2):212-8
21. McDonald SD, Yu ZM, van Blyderveen S, Schmidt L, Sword W, Vanstone $\mathrm{M}$, et al. Prediction of excess pregnancy weight gain using psychological, physical, and social predictors: a validated model in a prospective cohort study. PLoS One. 2020;15(6):e0233774.

22. Novaes ES, Melo EC, Ferracioli PL, Oliveira RR, Mathias TA. Gestational risk and associated factors in women cared by the public health network. Cienc Cuid Saude. 2018,17(3):e45232.

23. World Health Organization (WHO). Obesity among women. Geneva: WHO; 2017 [cited 5 Dec 2017]. Available from: http://www.who.int/ gho/urban_health/physiological-risk-factors/women_obesity/en/

24. Johnson JL, Farr SL, Dietz PM, Sharma AJ, Barfield WD, Robbins $\mathrm{CL}$. Trends in gestational weight gain: the Pregnancy Risk Assessment Monitoring System, 2000-2009. Am J Obstet Gynecol. 2015;212(6):806.e1-8.

25. Glazer Kb, Danilack VA, Werner EF, Field AE, Savitz DA. Elucidating the role of overweight and obesity in racial and ethnic disparities in cesarean delivery risk. Annals Epidemiology. 2020;42:4-11.e4.

26. Phelan S, Abrams B, Wing RR. Prenatal intervention with partial meal replacement improves micronutrient intake of pregnant women with obesity. Nutrients. 2019;11(5):1071

27. Szumilewicz A. Who and how should prescribe and conduct exercise programs for pregnant women? Recommendation based on the European educational standards for pregnancy and postnatal exercise specialists [Editorial]. Dev Period Med. 2018;22(2):107-12.

28. Coelho NL, Cunha DB, Esteves AP, Lacerda EM, Theme Filha MM. Dietary patterns in pregnancy and birth weight. Rev Saude Publica. 2015;49(62):49-62.

29. Oliveira AC, Tavares MC, Bezerra AR. Eating insecurity among pregnant women in the public health system in a state capital in the northeast of Brazil. Cien Saude Colet. 2017;22(2):519-26.

30. Teixeira CS, Cabral AC. Avaliação nutricional de gestantes sob acompanhamento em serviços de pré-natal distintos: a região metropolitana e 0 ambiente rural. Rev Bras Ginecol Obstet. 2016;8(1):27-34

31. Cheikh Ismail L, Bishop DC, Pang R, Ohuma EO, Kac G, Abrams B, et al. Gestational weight gain standards based on women enrolled in the Fetal Growth Longitudinal Study of the INTERGROWTH-21st Project: a prospective longitudinal cohort study. BMJ. 2016;352:i555.

32. Silva CS, Souza KV, Alves VH, Cabrita BA, Silva LR. Atuação do enfermeiro na consulta pré-natal: limites e potencialidades. Rev Pesa Cuid Fundam Online. 2016;8(2):4087-98.

33. Marques BL, Tomasi YT, Saraiva SS, Boing AF, Geremia DS. Guidelines to pregnant women: the importance of the shared care in primary health care. Esc Anna Nery. 2020;25(1):e20200098.

34. Bruno R, Petrella E, Bertarini V, Pedrielli G, Neri I, Facchinetti F. Adherence to a lifestyle programme in overweight/obese pregnant women and effect on gestational diabetes mellitus: a randomized controlled trial. Matern Child Nutr. 2017;13(3):e12333.

35. Muktabhant B, Lawrie TA, Lumbiganon P, Laopaiboon M. Diet or exercise, or both, for preventing excessive weight gain in pregnancy. Cochrane Database Syst Rev. 201515;(6):CD007145. Review.

36. Sampaio LM, Reis AP, Neves GA, Andrade DL. Street network: importance of prenatal follow-up and linking of pregnant to reference maternity. Cienc Cuid Saude. 2018;17(1):e-38384.

37. Hunter A, Devane D, Houghton C, Grealish A, Tully A, Smith V. Womancentred care during pregnancy and birth in Ireland: thematic analysis of women's and clinicians' experiences. BMC Pregnancy Childbirth. 2017;17(1):322. 